\title{
A Convenient Method for the Preparation of
}

\author{
Weinreb Amides via Pd-Catalyzed
}

\section{Aminocarbonylation of Aryl Bromides at}

\author{
Atmospheric Pressure
}

Joseph R Martinelli, Dominique M. M. Freckmann and Stephen L. Buchwald*

Department of Chemistry, Massachusetts Institute of Technology

Cambridge, Massachusetts, 02139.

\section{Experimental Section}

General. All reactions were carried out under a carbon monoxide atmosphere, Air Gas. Elemental analyses were performed by Atlantic Microlabs Inc., Norcross, GA. Unless otherwise noted, THF, $\mathrm{Et}_{2} \mathrm{O}, \mathrm{CH}_{2} \mathrm{Cl}_{2}$ and toluene were purchased from J.T. Baker in CYCLE-TAINER ${ }^{\circledR}$ solvent-delivery kegs and vigorously purged with argon for $2 \mathrm{~h}$. The solvents were further purified by passing them under argon pressure through two packed columns of neutral alumina (for THF and $\mathrm{Et}_{2} \mathrm{O}$ ) or through neutral alumina and copper (II) oxide (for toluene and $\mathrm{CH}_{2} \mathrm{Cl}_{2}$ ). ${ }^{1}$ Unless otherwise stated, commercially obtained materials were used without further purification. 
The following aryl bromides were purchased form Acros: 3-bromothiophene (filtered through basic alumina prior to use) and 2-bromo-3-methylpyridine. The following aryl bromides were purchased from Lancaster: 3-bromonitrobenzene, 4-bromo-2-fluorobenzonitrile, methyl 3bromobenzoate and 1-bromo-2-cyclohexylbenzene. The following aryl bromides were purchased from Alfa Aesar: 3-bromobenzonitrile (Avocado Organics), 4-chloro-bromobenzene (Avocado Organics), 4-bromoanisole (filtered through basic alumina prior to use), 1-bromonaphthalene (Avocado Organics), 2-bromobenzonitrile, 2-bromobenzotrifluoride (filtered through basic alumina prior to use) and 2-bromoanisole (Avocado Organics; filtered through basic alumina prior to use). The following aryl bromides were purchased from Aldrich: 4-bromoveratrole (filtered through basic alumina prior to use), 4-bromobiphenyl, 2-(3-bromophenyl)-1,3-dioxolane (filtered through basic alumina prior to use), 2-bromo-p-xylene, methyl 2-bromobenzoate and 4chlorobenzonitrile. The following compound was purchased from PCR Inc.: 2,5difluorobromobenzene (filtered through basic alumina prior to use). tert-Butyl $N$-(4bromophenyl)carbamate was prepared following literature procedures ${ }^{2}$ using 4-bromoaniline (Aldrich), Di-tert-butyl dicarbonate (Aldrich) and Iodomethane (Alfa). $N, \quad O-$ dimethylhydroxylamine hydrochloride was purchased from Aldrich and Alfa Aesar. Xantphos was purchased form Strem and used without further purification. $\operatorname{Pd}(\mathrm{OAc})_{2}$ was purchased from Strem, Inc. or supplied by Englehard. Sodium Carbonate was purchased from Mallinckrodt. Anhydrous tribasic potassium phosphate was purchased from Fluka Chemical Co. and used as supplied. The source (and thus the particle size) of the base employed may be critical for achieving efficient reactions.

All products of aminocarbonylation reactions were characterized by ${ }^{1} \mathrm{H}$ NMR, ${ }^{13} \mathrm{C}$ NMR, and IR spectroscopy, as well as elemental analysis (Atlantic Microlab, Inc). Two new compounds 
failed to give satisfactory elemental analyses. For these copies of ${ }^{1} \mathrm{H}$ and ${ }^{13} \mathrm{C}$ NMR spectra are included. Nuclear Magnetic Resonance spectra were recorded on a Varian Mercury 300 Infrared spectra were recorded using a Perkin-Elmer 2000 FT-IR. All ${ }^{1} \mathrm{H}$ NMR experiments are reported in $\delta$ units, parts per million (ppm) downfield from tetramethylsilane (internal standard) and were measured relative to the signals for residual chloroform (7.26 ppm), methylene chloride $(5.32 \mathrm{ppm})$ or benzene $(7.16 \mathrm{ppm})$ in the deuterated solvents. All ${ }^{13} \mathrm{C}$ NMR spectra are reported in ppm relative to deuterochloroform $(77.23 \mathrm{ppm})$, deuteromethylene chloride $(54.00 \mathrm{ppm})$ or deuterobenzene (128.39 ppm), and all were obtained with ${ }^{1} \mathrm{H}$ decoupling. All ${ }^{31} \mathrm{P}$ NMR spectra are reported in ppm relative to $\mathrm{H}_{3} \mathrm{PO}_{4}(0 \mathrm{ppm})$. All ${ }^{19} \mathrm{~F}$ NMR spectra are reported in ppm relative to trichlorofluoromethane (0 ppm). Melting points (uncorrected) were obtained on a Mel-Temp capillary melting point apparatus. Gas Chromatographic analyses were performed on a HewlettPackard 6890 gas chromatography instrument with an FID detector using $25 \mathrm{~m} \mathrm{x} 0.20 \mathrm{~mm}$ capillary column with cross-linked methyl siloxane as a stationary phase.

The conversions in Table 1 were determined by G.C. using dodecane as an internal standard, added during reaction workup. The yields in Table 1, entries $1-9$ were also determined by G.C. using dodecane as an internal standard. The yield in Table 1, entry 10, is an isolated yield (average of two runs) and the procedure is given below. The yields in Tables 2 and 3 are isolated yields (average of two runs). All compounds isolated were estimated to be $\geq 95 \%$ pure as determined by ${ }^{1} \mathrm{H}$ NMR and GC analysis and/or combustion analysis. The procedures described in this section are representative, and thus the yields may differ from those shown in Tables 1 - 3 .

\section{Synthesis of Weinreb Amides}

General Procedure A: Synthesis of Wienreb Amides via Pd-Catalyzed Aminocarbonylation. 
An oven-dried culture tube $(18 \times 150 \mathrm{~mm}$, VWR $)$ equipped with a Teflon ${ }^{\circledR}$ coated magnetic stir bar was sealed with a 14/20 rubber septum (inverted), evacuated, backfilled with nitrogen and cooled under nitrogen. All solid reagents were added by briefly removing the rubber septum: $\mathrm{Pd}(\mathrm{OAc})_{2}(2 \mathrm{~mol} \%, 0.02 \mathrm{mmol}, 0.02$ equiv., $4.5 \mathrm{mg})$, Xantphos $(2 \mathrm{~mol} \%, 0.02$ mmol, 0.02 equiv., $11.6 \mathrm{mg}), N$, $O$-dimethylhydroxylamine hydrochloride (1.5 mmol, 1.5 equiv., $146 \mathrm{mg}$ ), and $\mathrm{Na}_{2} \mathrm{CO}_{3}$ ( $3 \mathrm{mmol}, 3$ equiv., $318 \mathrm{mg}$ ). Then, all liquid reagents were added dropwise via syringe: aryl bromide ( $1 \mathrm{mmol}, 1$ equiv.; aryl bromides which were solids at room temperature were added during the initial charge) and toluene $(2 \mathrm{~mL})$. After the addition of all reagents, the rubber septum was secured with several wrappings of electrical tape. Then, the reaction was purged for $\sim 30$ seconds with $\mathrm{CO}_{(\mathrm{g})}$; following the gas purge a balloon was connected to the reaction using a short length of rubber tubing $(\sim 1 \mathrm{in}$.), a needle adapter and a 20 $\mathrm{G}$ needle. This balloon was then inflated with $\mathrm{CO}_{(\mathrm{g})}$ and the reaction tube was submerged in a 80 ${ }^{\circ} \mathrm{C}$ preheated oil bath. The reaction mixture was heated at $80{ }^{\circ} \mathrm{C}$ with vigorous stirring until the aryl halide had been completely consumed as judged by GC analysis. The reaction mixture was then allowed to cool to room temperature, diluted with ethyl acetate $(\sim 10 \mathrm{~mL})$, filtered through a plug of celite (eluting with ethyl acetate) and concentrated under reduced pressure. The crude material obtained was purified by flash chromatography on silica gel.

\section{General Procedure B: Synthesis of Wienreb Amides via Pd-Catalyzed Aminocarbonylation of ortho-Substituted Aryl Halides.}

An oven-dried culture tube $(18$ x $150 \mathrm{~mm}, \mathrm{VWR})$ equipped with a Teflon ${ }^{\circledR}$ coated magnetic stir bar was sealed with a 14/20 rubber septum (inverted), evacuated, backfilled with nitrogen and cooled under nitrogen. All solid reagents were added by briefly removing the rubber septum: $\mathrm{Pd}(\mathrm{OAc})_{2}(2.5 \mathrm{~mol} \%, 0.025 \mathrm{mmol}, 0.025$ equiv., $5.6 \mathrm{mg})$, Xantphos $(5 \mathrm{~mol} \%$, 
$0.05 \mathrm{mmol}, 0.05$ equiv., $28.9 \mathrm{mg}), N, O$-dimethylhydroxylamine hydrochloride (1.5 mmol, 1.5 equiv., $146 \mathrm{mg}$ ), and $\mathrm{K}_{3} \mathrm{PO}_{4}$ ( $3 \mathrm{mmol}, 3$ equiv., $637 \mathrm{mg}$ ). Then, all liquid reagents were added dropwise via syringe: aryl bromide $(1 \mathrm{mmol}, 1$ equiv.; aryl bromides which were solids at room temperature were added during the initial charge) and solvent ( $2 \mathrm{~mL}$, toluene or $m$-xylene). After the addition of all reagents, the rubber septum was secured with several wrappings of electrical tape. Then, the reaction was purged for $\sim 30$ seconds with $\mathrm{CO}_{(\mathrm{g})}$; following the gas purge a balloon was connected to the reaction using a short length of rubber tubing ( $\sim 1 \mathrm{in}$.), a needle adapter and a $20 \mathrm{G}$ needle. This balloon was then inflated with $\mathrm{CO}_{(\mathrm{g})}$ and the reaction tube was submerged in a $100-120^{\circ} \mathrm{C}$ preheated oil bath. The reaction mixture was heated at $100-120{ }^{\circ} \mathrm{C}$ with vigorous stirring until the aryl halide had been completely consumed as judged by GC analysis. The reaction mixture was then allowed to cool to room temperature, diluted with ethyl acetate $(\sim 10 \mathrm{~mL})$, filtered through a plug of celite (eluting with ethyl acetate) and concentrated under reduced pressure. The crude material obtained was purified by flash chromatography on silica gel.

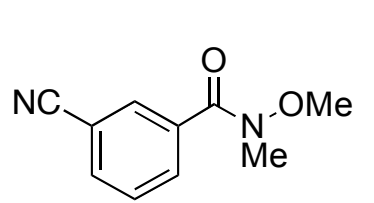

3-Cyano- $N$-methoxy- $N$-methyl-benzamide (Table 2, entry 1). Following general procedure $\mathrm{A}$, a mixture of 3-bromobenzonitrile ( $1 \mathrm{mmol}, 0.182 \mathrm{~g}), \mathrm{Pd}(\mathrm{OAc})_{2}(2 \mathrm{~mol} \%, 0.02 \mathrm{mmol}, 0.02$ equiv., $4.5 \mathrm{mg})$, Xantphos (2 mol \%, $0.02 \mathrm{mmol}, 0.02$ equiv., $11.6 \mathrm{mg}$ ), $\mathrm{N}, \mathrm{O}$ dimethylhydroxylamine hydrochloride ( $1.5 \mathrm{mmol}, 1.5$ equiv., $146 \mathrm{mg})$, $\mathrm{Na}_{2} \mathrm{CO}_{3}(3 \mathrm{mmol}, 3$ equiv., $318 \mathrm{mg})$, and toluene $(2 \mathrm{~mL})$ was heated at $80{ }^{\circ} \mathrm{C}$ for 8 hours. The crude product mixture was purified by flash column chromatography on silica gel (50\% ethyl acetate in hexanes) to provide the title compound as a viscous light orange oil (181 $\mathrm{mg}, 95 \%)$. ${ }^{1} \mathrm{H}$ NMR $\left(300 \mathrm{MHz}, \mathrm{CDCl}_{3}\right) \delta: 8.04-8.01(\mathrm{~m}, 1 \mathrm{H}), 7.97-7.93(\mathrm{dm}, J$ for the $\mathrm{d}=7.98 \mathrm{~Hz}, 1 \mathrm{H})$, 7.78-7.73 (dm, $J$ for the $\mathrm{d}=7.70 \mathrm{~Hz}, 1 \mathrm{H}), 7.55(\mathrm{ddd}, J=0.55,7.70,7.98 \mathrm{~Hz}, 1 \mathrm{H}), 3.54(\mathrm{~s}, 3 \mathrm{H})$, 3.39 (s, 3H). ${ }^{13} \mathrm{C}$ NMR $\left(75 \mathrm{MHz}, \mathrm{CDCl}_{3}\right) \delta: 167.06,135.02,133.74,132.48,131.84,128.93$, 118.01, 112.10, 61.19, 33.06. IR (neat, $\mathrm{cm}^{-1}$ ): 3075, 2975, 2938, 2821, 2232, 1647,1602, 1578, $1486,1460,1436,1412,1384,1178,986,799,734,684$. A satisfactory elemental analysis was not obtained for this compound: Anal. Calcd for $\mathrm{C}_{10} \mathrm{H}_{10} \mathrm{~N}_{2} \mathrm{O}_{2}: \mathrm{C}, 63.15 ; \mathrm{H}, 5.30$. Found: $\mathrm{C}$, 62.74; $\mathrm{H}, 5.22$. The ${ }^{1} \mathrm{H}$ and ${ }^{13} \mathrm{C}$ NMR spectra follow. 


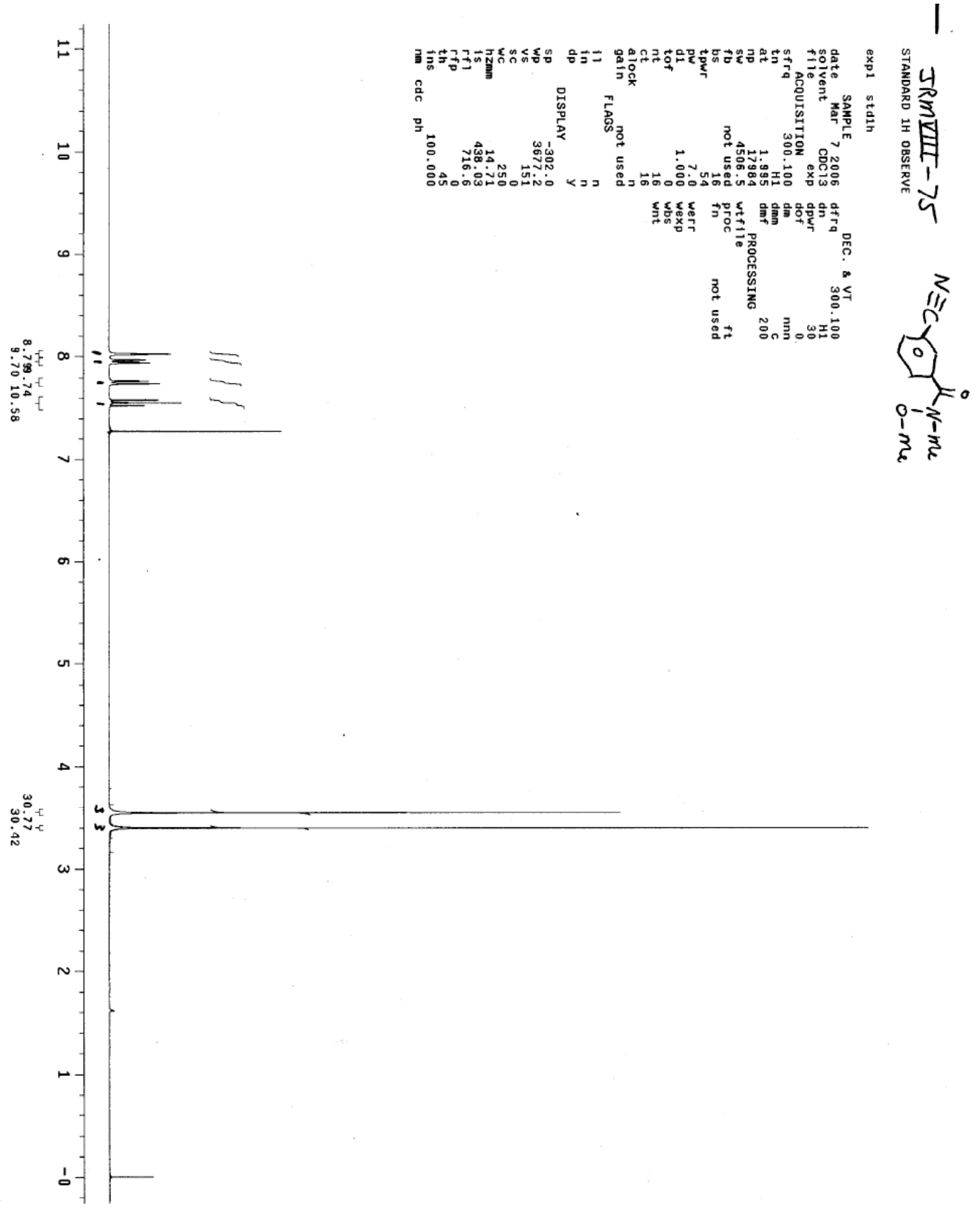




$$
1
$$


<smiles>COC(=O)c1ccc(C#N)c(F)c1</smiles>

4-Cyano-3-fluoro- $N$-methoxy- $N$-methyl-benzamide (Table 2 , entry 2). Following general procedure $\mathrm{A}$, a mixture of 4-bromo-2fluorobenzonitrile ( $1 \mathrm{mmol}, 0.200 \mathrm{~g}), \mathrm{Pd}(\mathrm{OAc})_{2}(2 \mathrm{~mol} \%, 0.02 \mathrm{mmol}$, 0.02 equiv., $4.5 \mathrm{mg})$, Xantphos ( $2 \mathrm{~mol} \%, 0.02 \mathrm{mmol}, 0.02$ equiv., 11.6 $\mathrm{mg}), \mathrm{N}, \mathrm{O}$-dimethylhydroxylamine hydrochloride $(1.5 \mathrm{mmol}, 1.5$ equiv., $146 \mathrm{mg}), \mathrm{Na}_{2} \mathrm{CO}_{3}(3 \mathrm{mmol}, 3$ equiv., $318 \mathrm{mg})$, and toluene $(2 \mathrm{~mL})$ was heated at $80{ }^{\circ} \mathrm{C}$ for 18 hours. The crude product mixture was purified by flash column chromatography on silica gel (20\% - 50\% ethyl acetate in hexanes) to provide the title compound as a light yellow-orange solid (181 mg, $95 \%$ ), mp 43 - $44{ }^{\circ} \mathrm{C}$. ${ }^{1} \mathrm{H}$ NMR (300 MHz, $\mathrm{CDCl}_{3}$ ) 8: 7.71-7.66 (m, 1H), 7.60-

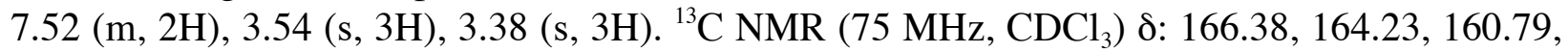
140.84, 140.74, 133.36, 124.70, 124.65, 116.64, 116.35, 113.42, 103.26, 103.06, 61.59, 33.17 (observed complexity due to C-F splitting; definitive assignments have not yet been made). ${ }^{19} \mathrm{~F}$

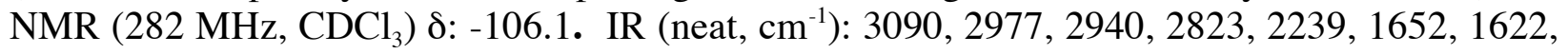
1566, 1503, 1459, 1428, 1386, 1251, 1198, 1182, 1115, 990, 941, 887, 835, 750, 733, 714, 682, 668. Anal. Calcd for $\mathrm{C}_{10} \mathrm{H}_{9} \mathrm{FN}_{2} \mathrm{O}_{2}$ : C, 57.69; H, 4.36. Found: C, 57.64; H, 4.37.<smiles>CON(C)C(=O)c1cccc([N+](=O)[O-])c1</smiles>

3-Nitro- $N$-methoxy- $N$-methyl-benzamide (Table 2, entry 3). Following general procedure $\mathrm{A}$, a mixture of 3-bromonitrobenzene $(1 \mathrm{mmol}, 0.202 \mathrm{~g}), \mathrm{Pd}(\mathrm{OAc})_{2}(2 \mathrm{~mol} \mathrm{\%}, 0.02 \mathrm{mmol}, 0.02$ equiv., $4.5 \mathrm{mg})$, Xantphos (2 mol \%, $0.02 \mathrm{mmol}, 0.02$ equiv., $11.6 \mathrm{mg}), \mathrm{N}, \mathrm{O}$ dimethylhydroxylamine hydrochloride ( $1.5 \mathrm{mmol}, 1.5$ equiv., $146 \mathrm{mg}$ ), $\mathrm{Na}_{2} \mathrm{CO}_{3}$ (3 mmol, 3 equiv., $318 \mathrm{mg}$ ), and toluene $(2 \mathrm{~mL})$ was heated at $80{ }^{\circ} \mathrm{C}$ for 8 hours. The crude product mixture was purified by flash column chromatography on silica gel (50\% ethyl acetate in hexanes) to provide the title compound as a tan colored solid (185 $\mathrm{mg}, 88 \%), \mathrm{mp} 41-$ $43{ }^{\circ} \mathrm{C} .{ }^{1} \mathrm{H}$ NMR $\left(300 \mathrm{MHz}, \mathrm{CDCl}_{3}\right) \delta: 8.60-8.58(\mathrm{t}, J=19 \mathrm{~Hz}, 1 \mathrm{H}), 8.35-8.31$ (ddd, $J=1.1,2.5$, 8.2 Hz, 1H), 8.07-8.03 (dt, $J=1.4,7.7 \mathrm{~Hz}, 1 \mathrm{H}), 7.65-7.59$ (t, $J=8 \mathrm{~Hz}, 1 \mathrm{H}), 3.57$ (s, 3H), 3.41 (s, $3 \mathrm{H}) .{ }^{13} \mathrm{C} \mathrm{NMR}\left(75 \mathrm{MHz}, \mathrm{CDCl}_{3}\right) \delta: 167.02,147.62,135.43,134.29,129.22,125.17,123.41$, 61.31, 33.13. IR (neat, $\mathrm{cm}^{-1}$ ): 3087, 2974, 2938, 2822, 1648, 1616, 1577, 1532, 1485, 1459, $1438,1417,1383,1351,1215,1170,1099,983,918,858,815$, 715. Anal. Calcd for $\mathrm{C}_{9} \mathrm{H}_{10} \mathrm{~N}_{2} \mathrm{O}_{4}$ : C, 51.43; H, 4.80. Found: C, 51.43; H, 4.70.

Thiophene-3- $N$-methoxy- $N$-methyl carboxamide (Table 2, entry 4).<smiles>CON(C)C(=O)c1ccsc1</smiles>
Following general procedure A, a mixture of 3-bromothiophene (1mmol, $0.163 \mathrm{~g}, 94 \mu \mathrm{L}$, filtered through basic alumina prior to use $), \mathrm{Pd}(\mathrm{OAc})_{2}(2 \mathrm{~mol}$ $\%, 0.02$ mmol, 0.02 equiv., $4.5 \mathrm{mg}$ ), Xantphos (2 mol \%, $0.02 \mathrm{mmol}, 0.02$ equiv., $11.6 \mathrm{mg}), \mathrm{N}, O$-dimethylhydroxylamine hydrochloride (1.5 mmol, 1.5 equiv., $146 \mathrm{mg}$ ), $\mathrm{Na}_{2} \mathrm{CO}_{3}\left(3 \mathrm{mmol}, 3\right.$ equiv., $318 \mathrm{mg}$ ), and toluene $(2 \mathrm{~mL})$ was heated at $80{ }^{\circ} \mathrm{C}$ for 21 hours. The crude product mixture was purified by flash column chromatography on silica gel (50\% ethyl acetate in hexanes) to provide the title compound as a very light yellow oil $(155 \mathrm{mg}, 90 \%) .{ }^{1} \mathrm{H}$ NMR (300 MHz, $\left.\mathrm{CDCl}_{3}\right) \delta: 8.09-8.06(\mathrm{dd}, J=1.1,3.0 \mathrm{~Hz}, 1 \mathrm{H}), 7.59-7.57(\mathrm{dd}, J=1.1,5.1,1 \mathrm{H})$, 7.31-7.27 (dd, $J=3.0,5.2 \mathrm{~Hz}, 1 \mathrm{H}), 3.66(\mathrm{~s}, 3 \mathrm{H}), 3.37(\mathrm{~s}, 3 \mathrm{H}) .{ }^{13} \mathrm{C} \mathrm{NMR}\left(75 \mathrm{MHz}, \mathrm{CDCl}_{3}\right) \delta$ : $163.57,134.37,130.79,128.95,124.77,61.10,33.17$. IR (neat, $\mathrm{cm}^{-1}$ ): 3109, 2970, 2936, 2819, 1627, 1518, 1458, 1427, 1387, 1350, 1217, 1182, 1153, 1078, 985, 931, 881, 851, 816, 792, 733, 707, 667, 621. Anal. Calcd for $\mathrm{C}_{7} \mathrm{H}_{9} \mathrm{NO}_{2} \mathrm{~S}$ : C, 49.10; H, 5.30. Found: C, 49.40; H, 5.40. 
<smiles>CN(C)C(=O)c1ccc(Cl)cc1</smiles>

4-Chloro- $N$-methoxy- $N$-methyl-benzamide (Table 2, entry 5). Following general procedure $\mathrm{A}$, a mixture of 4-chloro-bromobenzene ( $1 \mathrm{mmol}, 0.191 \mathrm{~g}), \mathrm{Pd}(\mathrm{OAc})_{2}(2 \mathrm{~mol} \%, 0.02 \mathrm{mmol}, 0.02$ equiv., $4.5 \mathrm{mg})$, Xantphos (2 mol \%, $0.02 \mathrm{mmol}, 0.02$ equiv., $11.6 \mathrm{mg}), N, O-$ dimethylhydroxylamine hydrochloride ( $1.5 \mathrm{mmol}, 1.5$ equiv., $146 \mathrm{mg}$ ), $\mathrm{Na}_{2} \mathrm{CO}_{3}(3 \mathrm{mmol}, 3$ equiv., $318 \mathrm{mg})$, and toluene $(2 \mathrm{~mL})$ was heated at $80{ }^{\circ} \mathrm{C}$ for 19 hours. The crude product mixture was purified by flash column chromatography on silica gel (50\% ethyl acetate in hexanes) to provide the title compound as a colorless oil (173 mg, $87 \%) .{ }^{1} \mathrm{H}$ NMR $\left(300 \mathrm{MHz}, \mathrm{CDCl}_{3}\right)$ 8: 7.79-7.64 (m, 2H), 7.41-7.36 (m, 2H), $3.54(\mathrm{~s}, 3 \mathrm{H}), 3.37(\mathrm{~s}, 3 \mathrm{H}) .{ }^{13} \mathrm{C}$ NMR $\left(75 \mathrm{MHz}, \mathrm{CDCl}_{3}\right) \delta: 168.53,136.62,132.27,129.82,128.21,61.06,33.43$. IR (neat, $\mathrm{cm}^{-1}$ ): 3067 , 2970, 2935, 2818, 1917, 1643, 1594, 1567, 1490, 1460, 1416, 1380, 1275, 1213, 1176, 1148, 1111, 1091, 1016, 995, 979, 887, 840, 746, 691, 656, 627. Anal. Calcd for $\mathrm{C}_{9} \mathrm{H}_{10} \mathrm{ClNO}_{2}$ : C, 54.15; H, 5.05. Found: C, 54.23; H, 4.92 .<smiles>CCCCC(=O)Nc1ccc(C(=O)N(C)C)cc1</smiles>

tert-butyl $\quad N$-methyl- $N$ - ( 4 - $\quad N$-methoxy- $N$-methylbenzamide)carbamate (Table 2 , entry 6). Following general procedure A, a mixture of tert-Butyl $N$-(4bromophenyl)carbamate (1mmol, $0.285 \mathrm{~g}), \mathrm{Pd}(\mathrm{OAc})_{2}(2 \mathrm{~mol} \%$, $0.02 \mathrm{mmol}, 0.02$ equiv., $4.5 \mathrm{mg}$ ), Xantphos ( $2 \mathrm{~mol} \%, 0.02 \mathrm{mmol}$, 0.02 equiv., $11.6 \mathrm{mg}), \mathrm{N}, O$-dimethylhydroxylamine hydrochloride ( $1.5 \mathrm{mmol}, 1.5$ equiv., $146 \mathrm{mg}$ ), $\mathrm{Na}_{2} \mathrm{CO}_{3}$ ( $3 \mathrm{mmol}, 3$ equiv., $318 \mathrm{mg}$ ), and toluene $(2 \mathrm{~mL})$ was heated at $80{ }^{\circ} \mathrm{C}$ for 13 hours. The crude product mixture was purified by flash column chromatography on silica gel (50\% ethyl acetate in hexanes) to provide the title compound as a light brown oil $(210 \mathrm{mg}, 95 \%) .{ }^{1} \mathrm{H}$ NMR $\left(300 \mathrm{MHz}, \mathrm{CDCl}_{3}\right)$ \&: 7.73-7.66 (m, 2H), 7.33-7.28 (m, 2H), $3.57(\mathrm{~s}, 3 \mathrm{H}), 3.37(\mathrm{~s}, 3 \mathrm{H}), 3.29(\mathrm{~s}, 3 \mathrm{H}), 1.48(\mathrm{~s}, 9 \mathrm{H}) .{ }^{13} \mathrm{C}$ NMR $(75 \mathrm{MHz}$, $\mathrm{CDCl}_{3}$ ) : $169.01,154.18,145.69,130.13,128.71,124.15,80.58,60.89,36.83,33.62,28.14$, 27.70. IR (neat, $\mathrm{cm}^{-1}$ ): 2976, 2934, 2819, 1791, 1703, 1644, 1607, 1569, 1512, 1477, 1456, 1422, 1367, 1315, 1300, 1279, 1254, 1216, 1153, 1109, 1065, 1018, 995, 977, 889, 851, 807, 770, 758, 734, 700. Anal. Calcd for $\mathrm{C}_{15} \mathrm{H}_{22} \mathrm{~N}_{2} \mathrm{O}_{4}$ : C, 61.21; H, 7.53. Found: C, 60.91; H, 7.75.<smiles>COC(=O)c1ccc(OC)cc1</smiles>
mmol, 1.5 equiv., $146 \mathrm{mg}), \mathrm{Na}_{2} \mathrm{CO}_{3}(3 \mathrm{mmol}, 3$ equiv., $318 \mathrm{mg})$, and toluene $(2 \mathrm{~mL})$ was heated at $80{ }^{\circ} \mathrm{C}$ for 8 hours. The crude product mixture was purified by flash column chromatography on silica gel (50\% ethyl acetate in hexanes) to provide the title compound as a colorless oil (210 $\mathrm{mg}, 95 \%)$. ${ }^{1} \mathrm{H}$ NMR $\left(300 \mathrm{MHz}, \mathrm{CDCl}_{3}\right)$ 8: 7.77-7.70 (m, 2H), 6.94-6.88 (m, 2H), $3.85(\mathrm{~s}, 3 \mathrm{H})$, 3.57 (s, 3H), $3.36(\mathrm{~s}, 3 \mathrm{H}) .{ }^{13} \mathrm{C} \mathrm{NMR}\left(75 \mathrm{MHz}, \mathrm{CDCl}_{3}\right)$ 8: 169.21, 161.39, 130.39, 125.84, 113.11, 60.73, 55.16, 33.74. IR (neat, $\left.\mathrm{cm}^{-1}\right): 3074,3002,2966,2936,2840,2559,2048,1639,1608$, $1575,1512,1462,1421,1375,1304,1255,1216,1173,1112,1064,1029,994,977,888,842$, 796, 756, 703, 676, 631, 593. Anal. Calcd for $\mathrm{C}_{10} \mathrm{H}_{13} \mathrm{NO}_{3}$ : C, 61.53; H, 6.71. Found: C, 61.29; H, 6.69 . 
<smiles>COCNC(=O)c1cccc(C(=O)OC)c1</smiles>

$N$-Methoxy- $N$-methyl-isophthalamic acid methyl ester (Table 2, entry 8). Following general procedure A, a mixture of methyl 3bromobenzoate $(1 \mathrm{mmol}, 0.215 \mathrm{~g}), \mathrm{Pd}(\mathrm{OAc})_{2}(2 \mathrm{~mol} \%, 0.02 \mathrm{mmol}$, 0.02 equiv., $4.5 \mathrm{mg})$, Xantphos ( $2 \mathrm{~mol} \%, 0.02 \mathrm{mmol}, 0.02$ equiv., $11.6 \mathrm{mg}), \mathrm{N}, \mathrm{O}$-dimethylhydroxylamine hydrochloride ( $1.5 \mathrm{mmol}, 1.5$ equiv., $146 \mathrm{mg}), \mathrm{Na}_{2} \mathrm{CO}_{3}\left(3 \mathrm{mmol}, 3\right.$ equiv., $318 \mathrm{mg}$ ), and toluene $(2 \mathrm{~mL})$ was heated at $80{ }^{\circ} \mathrm{C}$ for 24 hours. The crude product mixture was purified by flash column chromatography on silica gel (50\% ethyl acetate in hexanes) to provide the title compound as a colorless oil (196 mg, $88 \%$ ). ${ }^{1} \mathrm{H}$ NMR $\left(300 \mathrm{MHz}, \mathrm{CDCl}_{3}\right.$ ) $8: 8.38-8.33(\mathrm{t}, J=1.7 \mathrm{~Hz}, 1 \mathrm{H}), 8.17-8.10$ (ddd, $J=1.4,1.7,7.9 \mathrm{~Hz}$, $1 \mathrm{H}), 7.91-7.84(\mathrm{ddd}, J=1.4,1.7,7.7 \mathrm{~Hz}, 1 \mathrm{H}), 7.54-7.46(\mathrm{dt}, J=1.7,7.7 \mathrm{~Hz}, 1 \mathrm{H}), 3.94(\mathrm{~s}, 3 \mathrm{H})$,

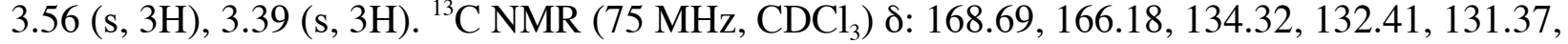
129.88, 129.19, 128.13, 60.99, 52.13, 33.30. IR (neat, $\mathrm{cm}^{-1}$ ): 3072, 2953, 1725, 1645, 1582, 1487, 1435, 1381, 1300, 1278, 1208, 1170, 1109, 1085, 991, 972, 922, 824, 772, 724, 665, 633, 575. Anal. Calcd for $\mathrm{C}_{11} \mathrm{H}_{13} \mathrm{NO}_{4}$ : C, 59.19; H, 5.87. Found: C, 58.98; H, 5.78.

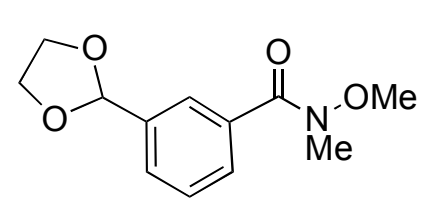

3-[1,3]Dioxolan-2-yl- $N$-methoxy- $N$-methyl-benzamide (Table 2, entry 9). Following general procedure A (a screw-capped test tube with a Teflon-lined septum was used in place of the culture tube and rubber septum), a mixture of 2-(3-bromophenyl)-1,3-dioxolane ( $1 \mathrm{mmol}, 151 \mathrm{~mL}$, filtered through basic alumina prior to use), $\mathrm{Pd}(\mathrm{OAc})_{2}(2 \mathrm{~mol} \%, 0.02 \mathrm{mmol}, 0.02$ equiv., $4.5 \mathrm{mg})$, Xantphos (2 mol \%, $0.02 \mathrm{mmol}, 0.02$ equiv., $11.6 \mathrm{mg}), \mathrm{N}, O$-dimethylhydroxylamine hydrochloride (1.5 mmol, 1.5 equiv., $146 \mathrm{mg}$ ), triethylamine ( $3 \mathrm{mmol}, 3$ equiv., $420 \mathrm{~mL})$, and toluene $(1 \mathrm{~mL})$ was heated at $80{ }^{\circ} \mathrm{C}$ for 15 hours. The crude product mixture was purified by flash column chromatography on silica gel (67\% ethyl acetate in hexanes) to provide the title compound as a colorless oil $(220 \mathrm{mg}, 93 \%) .{ }^{1} \mathrm{H}$ NMR (400 MHz, CDCl 3 ) $7.78(\mathrm{~s}, 1 \mathrm{H}), 7.67(\mathrm{~d}, 1 \mathrm{H}, J=7.5 \mathrm{~Hz}), 7.56(\mathrm{~d}, 1 \mathrm{H}, J=7.5 \mathrm{~Hz}), 7.41$ $(\mathrm{dd}, 1 \mathrm{H}, J=7.5 \mathrm{~Hz}), 5.83(\mathrm{~s}, 1 \mathrm{H}), 4.00-4.14(\mathrm{~m}, 4 \mathrm{H}), 3.53(\mathrm{~s}, 3 \mathrm{H}), 3.33(\mathrm{~s}, 3 \mathrm{H}) .{ }^{13} \mathrm{C} \mathrm{NMR}$ $\left(100 \mathrm{MHz}, \mathrm{CDCl}_{3}\right)$ d 169.4, 138.0, 134.1, 128.8, 128.6, 128.0, 126.3, 103.1, 65.2, 60.9, 33.6. IR $\left(\mathrm{CDCl}_{3}, \mathrm{~cm}^{-1}\right)$ 2972, 2937, 2892, 1639. Anal. Cald. for $\mathrm{C}_{12} \mathrm{H}_{15} \mathrm{NO}_{4}$; C: 60.75, $\mathrm{H}: 6.37$; Found C: $60.37, \mathrm{H}: 6.35$.<smiles>COc1ccc(C(=O)N(C)OC)cc1OC</smiles>

3,4, $N$-Trimethoxy- $N$-methyl-benzamide (Table 2 , entry 10 ). Following general procedure A (a screw-capped test tube with a Teflonlined septum was used in place of the culture tube and rubber septum), a mixture of 4-bromoveratrole ( $1 \mathrm{mmol}, 144 \mu \mathrm{L}$, filtered through basic alumina prior to use $), \mathrm{Pd}(\mathrm{OAc})_{2}(2 \mathrm{~mol} \%, 0.02 \mathrm{mmol}, 0.02$ equiv., 4.5 $\mathrm{mg}$ ), Xantphos (2 mol \%, $0.02 \mathrm{mmol}, 0.02$ equiv., $11.6 \mathrm{mg}), \mathrm{N}, \mathrm{O}$ dimethylhydroxylamine hydrochloride ( $1.5 \mathrm{mmol}, 1.5$ equiv., $146 \mathrm{mg}$ ), triethylamine ( $3 \mathrm{mmol}, 3$ equiv., $420 \mu \mathrm{L})$, and toluene $(1 \mathrm{~mL})$ was heated at $80{ }^{\circ} \mathrm{C}$ for 15 hours. The crude product mixture was purified by flash column chromatography on silica gel (67\% ethyl acetate in hexanes) to provide the title compound as a colorless solid $(202 \mathrm{mg}, 90 \%), \mathrm{mp} 55-57{ }^{\circ} \mathrm{C} .{ }^{1} \mathrm{H}$ NMR $\left(400 \mathrm{MHz}, \mathrm{CDCl}_{3}\right) 7.39(\mathrm{dd}, 1 \mathrm{H}, J=2 \mathrm{~Hz}, 8 \mathrm{~Hz}), 7.32(\mathrm{~d}, 1 \mathrm{H}, J=2 \mathrm{~Hz}), 6.87(\mathrm{~d}, 1 \mathrm{H}, J=$ $8 \mathrm{~Hz}), 3.92(\mathrm{~s}, 3 \mathrm{H}), 3.91(\mathrm{~s}, 3 \mathrm{H}), 3.58(\mathrm{~s}, 3 \mathrm{H}), 3.36(\mathrm{~s}, 3 \mathrm{H}) \cdot{ }^{13} \mathrm{C}$ NMR $\left(100 \mathrm{MHz}, \mathrm{CDCl}_{3}\right) \mathrm{d}$ 168.9, 150.8, 148.0, 125.9, 121.8, 111.7, 109.9, 60.7, 55.7, 55.6, 33.7. IR $\left(\mathrm{CDCl}_{3}, \mathrm{~cm}^{-1}\right)$ 2966, 2937, 1631, 1517. m. p. 56 - $57{ }^{\circ}$ C. Anal. Cald. for $\mathrm{C}_{11} \mathrm{H}_{15} \mathrm{NO}_{4}$; C: 58.66, H: 6.71; Found C: 58.61, H: 6.77. 
Biphenyl-4- $N$-methoxy- $N$-methyl carboxamide (Table 2, entry 11). ${ }^{3}$ Me Following general procedure A (a screw-capped test tube with a Teflonlined septum was used in place of the culture tube and rubber septum), a mixture of 4-bromobiphenyl ( $1 \mathrm{mmol}, 0.233 \mathrm{~g}), \mathrm{Pd}(\mathrm{OAc})_{2}(2 \mathrm{~mol} \%, 0.02$ mmol, 0.02 equiv., $4.5 \mathrm{mg}$ ), Xantphos ( $2 \mathrm{~mol} \%, 0.02 \mathrm{mmol}, 0.02$ equiv., $11.6 \mathrm{mg}), N, O$-dimethylhydroxylamine hydrochloride $(1.5 \mathrm{mmol}, 1.5$ equiv., $146 \mathrm{mg})$, triethylamine ( $3 \mathrm{mmol}, 3$ equiv., $420 \mu \mathrm{L}$ ), and toluene $(1 \mathrm{~mL})$ was heated at $80{ }^{\circ} \mathrm{C}$ for 15 hours. The crude product mixture was purified by flash column chromatography on silica gel (40\% ethyl acetate in hexanes) to provide the title compound as a colorless solid (226 mg, 94\%), mp $80-82{ }^{\circ} \mathrm{C}$, lit. mp $77-78{ }^{\circ} \mathrm{C} .{ }^{1} \mathrm{H}$ NMR (400 $\mathrm{MHz}, \mathrm{CDCl}_{3}$ ) 7.77-7.81 (m, $\left.2 \mathrm{H}\right), 7.61-7.67$ (m, 4 $\mathrm{H}), 7.37-7.50(\mathrm{~m}, 3 \mathrm{H}), 3.61(\mathrm{~s}, 3 \mathrm{H}), 3.40(\mathrm{~s}, 3 \mathrm{H}) .{ }^{13} \mathrm{C} \mathrm{NMR}\left(100 \mathrm{MHz}, \mathrm{CDCl}_{3}\right) \delta$ 169.5, 143.2, 140.0, 132.7, 128.8, 128.7, 127.8, 127.1, 126.6, 61.0, 33.7. IR $\left(\mathrm{CDCl}_{3}, \mathrm{~cm}^{-1}\right)$ 2971, 2936, 1632.<smiles>CONC(=O)c1cc(C)ccc1C</smiles>

$N$-Methoxy-2,5, $N$-trimethyl-benzamide (Table 3, entry 1). Following general procedure $\mathrm{B}$, a mixture of 2-bromo- $p$-xylene ( $1 \mathrm{mmol}, 0.185 \mathrm{~g}$, $138 \mu \mathrm{L}), \mathrm{Pd}(\mathrm{OAc})_{2}(2.5 \mathrm{~mol} \%, 0.025 \mathrm{mmol}, 0.025$ equiv., $5.6 \mathrm{mg})$, Xantphos (5 mol \%, $0.05 \mathrm{mmol}, 0.05$ equiv., $28.9 \mathrm{mg}$ ), $N, O$ dimethylhydroxylamine hydrochloride ( $1.5 \mathrm{mmol}, 1.5$ equiv., $146 \mathrm{mg}$ ), $\mathrm{K}_{3} \mathrm{PO}_{4}(3 \mathrm{mmol}, 3$ equiv., $637 \mathrm{mg})$, and toluene $(2 \mathrm{~mL})$ was heated at $100{ }^{\circ} \mathrm{C}$ for 20 hours. The crude product mixture was purified by flash column chromatography on silica gel (50\% ethyl acetate in hexanes) to provide a mixture of rotamers (ratio not determined) of the title compound as a colorless oil $(166 \mathrm{mg}, 86 \%) .{ }^{1} \mathrm{H}$ NMR $\left(300 \mathrm{MHz}, \mathrm{CDCl}_{3}\right) \delta: 7.12-7.06(\mathrm{~m}, 3 \mathrm{H}), 3.56$ (brs, $3 \mathrm{H}$ ), 3.31 (brs, 3H), 2.32 (s, 3H), 2.29 (s, 3H). ${ }^{13} \mathrm{C}$ NMR (75 MHz, $\mathrm{CDCl}_{3}$ ) $\delta: 169.8,134.5$, 133.9, 130.6, 129.2, 129.2, 129.0, 125.8, 59.9, 31.9, 19.8, 17.7. IR (neat, $\left.\mathrm{cm}^{-1}\right): 3018,2969$, 2932, 2818, 2736, 1903, 1844, 1651, 1612, 1577, 1502, 1459, 1422, 1375, 1287, 1242, 1181, 1157, 1126, 1062, 1041, 998, 980, 922, 887, 838, 816, 777, 746, 706, 694, 642, 597. Anal. Calcd for $\mathrm{C}_{11} \mathrm{H}_{15} \mathrm{NO}_{2}$ : C, 68.37; H, 7.82. Found: C, 68.28; H, 7.86.

Naphthalene-1- $N$-methoxy- $N$-methyl carboxamide (Table 3, entry 2).<smiles>CON(C)C(=O)c1cccc2ccccc12</smiles>
Following general procedure $\mathrm{B}$, a mixture of 1-bromonaphthalene $(1 \mathrm{mmol}$, $0.207 \mathrm{~g}, 139 \mu \mathrm{L}), \mathrm{Pd}(\mathrm{OAc})_{2}(2.5 \mathrm{~mol} \%, 0.025 \mathrm{mmol}, 0.025$ equiv., 5.6 $\mathrm{mg}$ ), Xantphos (5 mol \%, $0.05 \mathrm{mmol}, 0.05$ equiv., $28.9 \mathrm{mg}), \mathrm{N}, \mathrm{O}-$ dimethylhydroxylamine hydrochloride $(1.5 \mathrm{mmol}, 1.5$ equiv., $146 \mathrm{mg}), \mathrm{K}-$ ${ }_{3} \mathrm{PO}_{4}(3 \mathrm{mmol}, 3$ equiv., $637 \mathrm{mg})$, and toluene $(2 \mathrm{~mL})$ was heated at $100{ }^{\circ} \mathrm{C}$ for 20 hours. The crude product mixture was purified by flash column chromatography on silica gel (50\% ethyl acetate in hexanes) to provide a mixture of rotamers (ratio not determined) of the title compound as a light orange oil (208 mg, $97 \%) .{ }^{1} \mathrm{H}$ NMR $\left(300 \mathrm{MHz}, \mathrm{CDCl}_{3}\right)$ 8: 7.94-7.85 (m, 3H), 7.587.47 (m, 4H), 3.4 (brs, 6H). ${ }^{13} \mathrm{C}$ NMR $\left(75 \mathrm{MHz}, \mathrm{CDCl}_{3}\right.$ ) $\delta: 169.1,132.7,129.1,128.9,127.8$, 126.3, 125.3, 124.3, 124.2, 123.7, 60.5, 32.4. IR (neat, $\mathrm{cm}^{-1}$ ): 3280, 3056, 3005, 2971, 2935, 2817, 1947, 1820, 1651, 1592, 1580, 1508, 1474, 1439, 1422, 1374, 1266, 11232, 1183, 1167, $1102,1027,1014,975,891,865,801,779,740,697,647,629,580$. Anal. Calcd for $\mathrm{C}_{13} \mathrm{H}_{13} \mathrm{NO}_{2}$ : C, 72.54; H, 6.09. Found: C, 72.16; H, 6.12. 


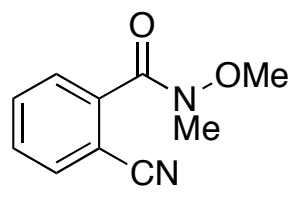

2-Cyano- $N$-methoxy- $N$-methyl-benzamide (Table 3, entry 3). Following general procedure $\mathrm{B}$, a mixture of 2-bromobenzonitrile (1mmol, $0.182 \mathrm{~g}$ ), $\mathrm{Pd}(\mathrm{OAc})_{2}(2.5 \mathrm{~mol} \%, 0.025 \mathrm{mmol}, 0.025$ equiv., $5.6 \mathrm{mg})$, Xantphos (5 mol $\%, 0.05$ mmol, 0.05 equiv., $28.9 \mathrm{mg}), \mathrm{N}, \mathrm{O}$-dimethylhydroxylamine hydrochloride ( $1.5 \mathrm{mmol}, 1.5$ equiv., $146 \mathrm{mg}), \mathrm{K}_{3} \mathrm{PO}_{4}(3 \mathrm{mmol}, 3$ equiv., 637 $\mathrm{mg})$, and toluene $(2 \mathrm{~mL})$ was heated at $100{ }^{\circ} \mathrm{C}$ for 20 hours. The crude product mixture was purified by flash column chromatography on silica gel (50\% ethyl acetate in hexanes) to provide a mixture of rotamers (ratio not determined) the title compound as a colorless oil (161 mg, 84 \%). ${ }^{1} \mathrm{H}$ NMR $\left(300 \mathrm{MHz}, \mathrm{CDCl}_{3}\right.$ ) $\delta: 7.74-7.70$ (ddd, $\left.J=0.55,1.38,7.7 \mathrm{~Hz}, 1 \mathrm{H}\right), 7.69-7.50$ (m,

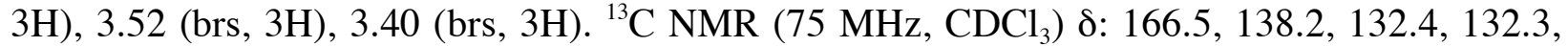
129.7, 127.3, 116.6, 109.9, 61.0, 32.3. IR (neat, $\mathrm{cm}^{-1}$ ): 3292, 3071, 2976, 2938, 2822, 2229, 1657, 1595, 1572, 1492, 1459, 1445, 1421, 1385, 1289, 1219, 1191, 1168, 1117, 1062, 1036, 982, 891, 772, 759, 720, 687, 634. Anal. Calcd for $\mathrm{C}_{10} \mathrm{H}_{10} \mathrm{~N}_{2} \mathrm{O}_{2}: \mathrm{C}, 63.15 ; \mathrm{H}, 5.30$. Found: C, 63.42; H, 5.29.

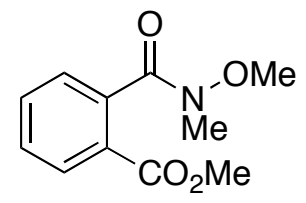

$N$-Methoxy- $N$-methyl-phthalamic acid methyl ester (Table 3, entry 4). Following general procedure $\mathrm{B}$, a mixture of methyl 2-bromobenzoate ( $1 \mathrm{mmol}, 0.215 \mathrm{~g}, 140 \mu \mathrm{L}), \mathrm{Pd}(\mathrm{OAc})_{2}(3.0 \mathrm{~mol} \%, 0.03 \mathrm{mmol}, 0.03$ equiv., 6.7 $\mathrm{mg}$ ), Xantphos (6 mol \%, 0.06 mmol, 0.06 equiv., $34.7 \mathrm{mg}), \mathrm{N}, \mathrm{O}-$ dimethylhydroxylamine hydrochloride ( $1.5 \mathrm{mmol}, 1.5$ equiv., $146 \mathrm{mg}), \mathrm{K}$ ${ }_{3} \mathrm{PO}_{4}(3 \mathrm{mmol}, 3$ equiv., $637 \mathrm{mg})$, and toluene $(2 \mathrm{~mL})$ was heated at $100{ }^{\circ} \mathrm{C}$ for 20 hours. The crude product mixture was purified by flash column chromatography on silica gel (20 - $50 \%$ ethyl acetate in hexanes) to provide the title compound as a colorless oil $(186 \mathrm{mg}, 83 \%) .{ }^{1} \mathrm{H}$

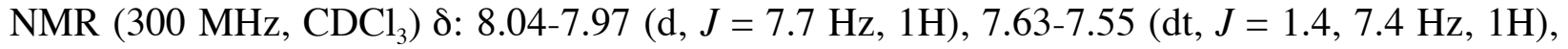
7.52-7.45 (dt, $J=1.4,7.7 \mathrm{~Hz}, 1 \mathrm{H}), 7.45-7.38(\mathrm{~d}, J=7.4 \mathrm{~Hz}), 3.91$ (s, 3H), 3.74 (brs, $3 \mathrm{H}), 3.35$ (brs, 3H). ${ }^{13} \mathrm{C}$ NMR $\left(75 \mathrm{MHz}, \mathrm{CDCl}_{3}\right.$ ) $\delta: 172.0,166.1,137.2,132.3,129.7,128.9,127.7,126.9$, 60.9, 52.4, 33.1. IR (neat, $\mathrm{cm}^{-1}$ ): 3067, 2953, 2939, 2904, 2820, 2845, 1726, 1662, 1599, 1578, 1492, 1459, 1413, 1435, 1379, 1280, 1211, 1192, 1166, 1130, 1091, 1062, 1040, 991, 964, 883, 828, 802, 777, 739, 723, 703, 667, 631, 576. Anal. Calcd for $\mathrm{C}_{11} \mathrm{H}_{13} \mathrm{NO}_{4}$ : C, 59.19; H, 5.87. Found: C, 59.17; H, 5.90.

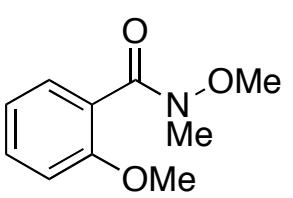

2,N-Dimethoxy- $N$-methyl-benzamide (Table 3, entry 5). ${ }^{4}$ Following general procedure $\mathrm{B}$, a mixture of 2-bromoanisole (1mmol, $0.187 \mathrm{~g}, 125 \mu \mathrm{L}$, filtered through basic alumina prior to use $), \operatorname{Pd}(\mathrm{OAc})_{2}(3.0 \mathrm{~mol} \%, 0.03$ mmol, 0.03 equiv., $6.7 \mathrm{mg}$ ), Xantphos (6 mol \%, $0.06 \mathrm{mmol}, 0.06$ equiv., $34.7 \mathrm{mg}), N, O$-dimethylhydroxylamine hydrochloride $(1.5 \mathrm{mmol}, 1.5$ equiv., $146 \mathrm{mg}), \mathrm{K}_{3} \mathrm{PO}_{4}(3 \mathrm{mmol}, 3$ equiv., $637 \mathrm{mg})$, and toluene $(2 \mathrm{~mL})$ was heated at $100{ }^{\circ} \mathrm{C}$ for 20 hours. The crude product mixture was purified by flash column chromatography on silica gel (50\% ethyl acetate in hexanes) to provide a mixture of rotamers (ratio not determined) of the title compound as a colorless plates $(174 \mathrm{mg}, 89 \%)$, mp $47-49{ }^{\circ} \mathrm{C} .{ }^{1} \mathrm{H} \mathrm{NMR}\left(300 \mathrm{MHz}, \mathrm{CDCl}_{3}\right)$ $\delta: 7.40-7.32$ (ddd, $J=1.6,7.4,8.2 \mathrm{~Hz}, 1 \mathrm{H}), 7.30-7.24(\mathrm{dd}, J=1.4,7.4 \mathrm{~Hz}, 1 \mathrm{H}), 7.01-6.95$ (dt, $J$ $=0.8,7.4 \mathrm{~Hz}, 1 \mathrm{H}), 6.95-6.90(\mathrm{~d}, J=8.3 \mathrm{~Hz}, 1 \mathrm{H}), 3.84(\mathrm{~s}, 3 \mathrm{H}), 3.49$ (brs, $3 \mathrm{H}), 3.33$ (brs, $3 \mathrm{H}) .{ }^{13} \mathrm{C}$

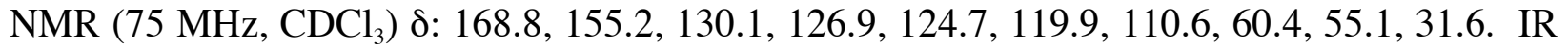
(neat, $\mathrm{cm}^{-1}$ ): 3067, 3003, 2970, 2938, 2939, 1651, 1601, 1584, 1495, 1465, 1437, 1418, 1381, 
1284, 1249, 1209, 1182, 1164, 1116, 1064, 1045, 1022, 987, 940, 884, 795, 758, 697, 630. Anal. Calcd for $\mathrm{C}_{10} \mathrm{H}_{13} \mathrm{NO}_{3}$ : C, 61.53; H, 6.71. Found: C, 61.46; H, 6.76.<smiles>CON(C)C(=O)c1ccc(C#N)cc1</smiles>

4-Cyano- $N$-methoxy- $N$-methyl-benzamide (Table 3 , entry 6). Following general procedure $\mathrm{B}$, a mixture of 4-chlorobenzonitrile (1mmol, $0.146 \mathrm{~g}), \mathrm{Pd}(\mathrm{OAc})_{2}(3.0 \mathrm{~mol} \%, 0.03 \mathrm{mmol}, 0.03$ equiv., 6.7 $\mathrm{mg}$ ), Xantphos (6 mol \%, $0.06 \mathrm{mmol}, 0.06$ equiv., $34.7 \mathrm{mg}$ ), $\mathrm{N}, \mathrm{O}$ dimethylhydroxylamine hydrochloride ( $1.5 \mathrm{mmol}, 1.5$ equiv., $146 \mathrm{mg}$ ), $\mathrm{K}_{3} \mathrm{PO}_{4}$ (3 mmol, 3 equiv., $637 \mathrm{mg}$ ), and toluene $(2 \mathrm{~mL})$ was heated at $105{ }^{\circ} \mathrm{C}$ for 20 hours. The crude product mixture was purified by flash column chromatography on silica gel (50\% ethyl acetate in hexanes) to provide the title compound as a colorless oil (149 mg, $78 \%)$. ${ }^{1} \mathrm{H}$ NMR

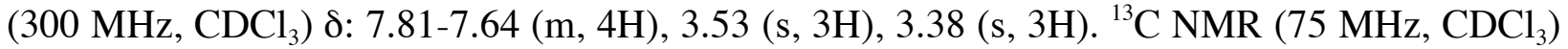
$\delta: 167.7,138.2,131.7,128.6,118.0,113.8,61.2,33.0$. IR (neat, $\mathrm{cm}^{-1}$ ): 3093, 3066, 2974, 2938, 2821, 2230, 1937, 1651, 1609, 1560, 1507, 1461, 1422, 1383, 1286, 1215, 1180, 1149, 1115, $1065,1020,980,889,851,7777,754,703,668,638,575$. Anal. Calcd for $\mathrm{C}_{10} \mathrm{H}_{10} \mathrm{~N}_{2} \mathrm{O}_{2}: \mathrm{C}, 63.15$; H, 5.30. Found: C, 63.12; H, 5.33.<smiles>CON(OC)C(=O)c1ccccc1C(F)(F)F</smiles>

2-trifluoromethyl- $N$-Methoxy- $N$-methy-benzamide (Table 3, entry 7). Following general procedure B, a mixture of 2-bromobenzotrifluoride (1mmol, $0.225 \mathrm{~g}, 136 \mu \mathrm{L}$, filtered through basic alumina prior to use), $\mathrm{Pd}(\mathrm{OAc})_{2}(3.0 \mathrm{~mol} \%, 0.03 \mathrm{mmol}, 0.03$ equiv., $6.7 \mathrm{mg})$, Xantphos (6 mol \%, $0.06 \mathrm{mmol}, 0.06$ equiv., $34.7 \mathrm{mg}), \quad N$, $O$-dimethylhydroxylamine hydrochloride ( $1.5 \mathrm{mmol}, 1.5$ equiv., $146 \mathrm{mg}), \mathrm{K}_{3} \mathrm{PO}_{4}(3 \mathrm{mmol}, 3$ equiv., $637 \mathrm{mg}$ ), and $m$-xylene $(2 \mathrm{~mL})$ was heated at $110{ }^{\circ} \mathrm{C}$ for 20 hours. The crude product mixture was purified by flash column chromatography on silica gel (20 - $50 \%$ ethyl acetate in hexanes) to provide the title compound as a colorless oil and a 1.1:1 mixture of rotamers $(214 \mathrm{mg}, 92 \%) .{ }^{1} \mathrm{H}$ NMR $(300$

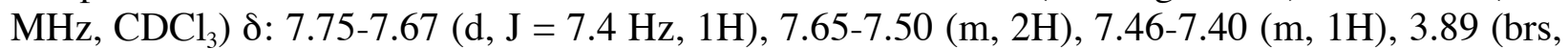
$0.6 \mathrm{H}), 3.42(\mathrm{~s}, 2.4 \mathrm{H}), 3.37(\mathrm{~s}, 2.3 \mathrm{H}), 3.05$ (brs, 0.7H). ${ }^{13} \mathrm{C} \mathrm{NMR}\left(75 \mathrm{MHz}, \mathrm{CDCl}_{3}\right) \delta: 169.1$, $164.4,133.7,132.1,131.3,129.5,128.9,127.2$, 126.8, 126.2, 1259, 125.3, 121.6, 118.0, 60.4, 59.7, 36.1, 31.9 (observed complexity due to C-F splitting; definitive assignments have not yet been made). ${ }^{19} \mathrm{~F}$ NMR (282 $\mathrm{MHz} \mathrm{CDCl}_{3}$ ) $\delta:-60.2$. IR (neat, $\left.\mathrm{cm}^{-1}\right): 3071,2977,2941,2823$, 1667, 1606, 1584, 1503, 1426, 1445, 1416, 1384, 1317, 1272, 1213, 1171, 1130, 1077, 1049, 1034, 991, 961, 891, 879, 772, 742, 708, 655, 632. Anal. Calcd for $\mathrm{C}_{10} \mathrm{H}_{10} \mathrm{~F}_{3} \mathrm{NO}_{2}$ : C, 51.51; $\mathrm{H}$, 4.32. Found: C, 51.61; H, 4.36 .

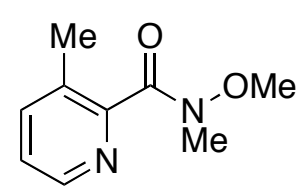

3-Methyl-pyridine-2- $N$-methoxy- $N$-methyl carboxamide (Table 3, entry 8). Following general procedure $\mathrm{B}$, a mixture of 2-bromo-3-methylpyridine (1mmol, $0.172 \mathrm{~g}, 111 \mu \mathrm{L}), \mathrm{Pd}(\mathrm{OAc})_{2}$ (3.0 mol \%, $0.03 \mathrm{mmol}, 0.03$ equiv., 6.7 $\mathrm{mg}$ ), Xantphos (6 mol \%, $0.06 \mathrm{mmol}, 0.06$ equiv., $34.7 \mathrm{mg}), \mathrm{N}, \mathrm{O}-$ dimethylhydroxylamine hydrochloride ( $1.5 \mathrm{mmol}, 1.5$ equiv., $146 \mathrm{mg}), \mathrm{K}-$ ${ }_{3} \mathrm{PO}_{4}(3 \mathrm{mmol}, 3$ equiv., $637 \mathrm{mg})$, and $m$-xylene $(2 \mathrm{~mL})$ was heated at $110{ }^{\circ} \mathrm{C}$ for 20 hours. The crude product mixture was purified by flash column chromatography on silica gel $(50-60 \%$ ethyl acetate in hexanes) to provide the title compound as a light yellow oil and a 1:1 mixture of rotamers (140 mg, $77 \%)$. ${ }^{1} \mathrm{H}$ NMR $\left(300 \mathrm{MHz} \mathrm{CDCl}_{3}\right) \delta: 8.44-8.36(\mathrm{dd}, \mathrm{J}=0.8,4.7 \mathrm{~Hz}, 1 \mathrm{H})$, 7.60-7.48 (d, J = 7.1 Hz, 1H), 7.27-7.16 (dd, J = 4.9, $7.7 \mathrm{~Hz}, 1 \mathrm{H}), 3.89$ (brs, 0.6H), 3.52 (s,

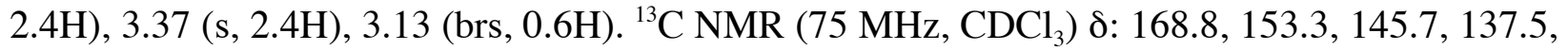


129.8, 123.4, 61.1, 31.4, 17.1. IR (neat, $\mathrm{cm}^{-1}$ ): 3055, 2977, 2938, 2821, 1655, 1575, 1485, 1446, 1407, 1384, 1274, 1260, 1238, 1186, 1169, 1119, 1072, 983, 896, 889, 818, 800, 743, 692, 639, 580. A satisfactory elemental analysis was not obtained for this compound: Anal. Calcd for $\mathrm{C}_{9} \mathrm{H}_{12} \mathrm{~N}_{2} \mathrm{O}_{2}$ : C, 59.99; H, 6.71. Found: C, 59.53; H, 6.72. The ${ }^{1} \mathrm{H}$ and ${ }^{13} \mathrm{C}$ NMR spectra follow. 


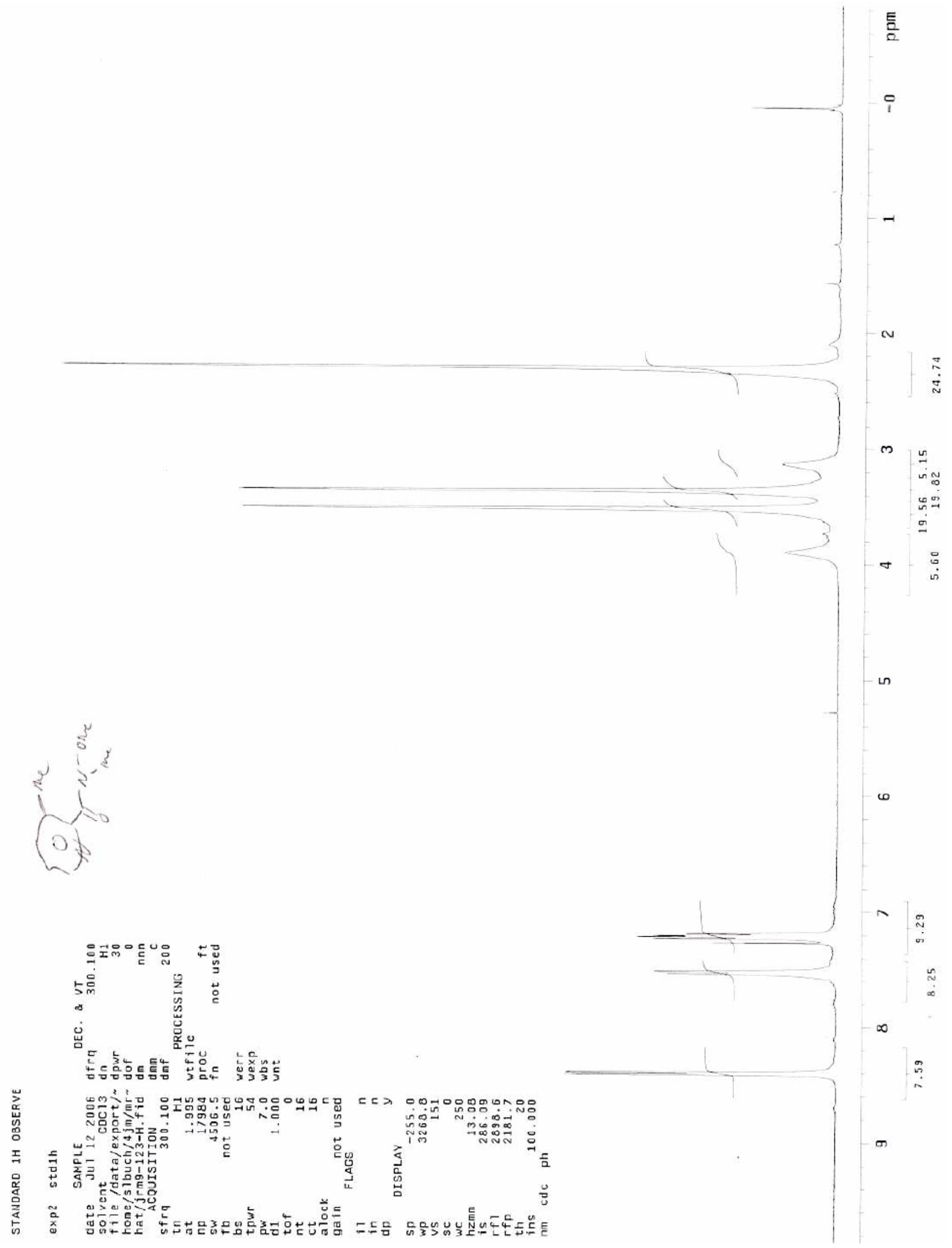




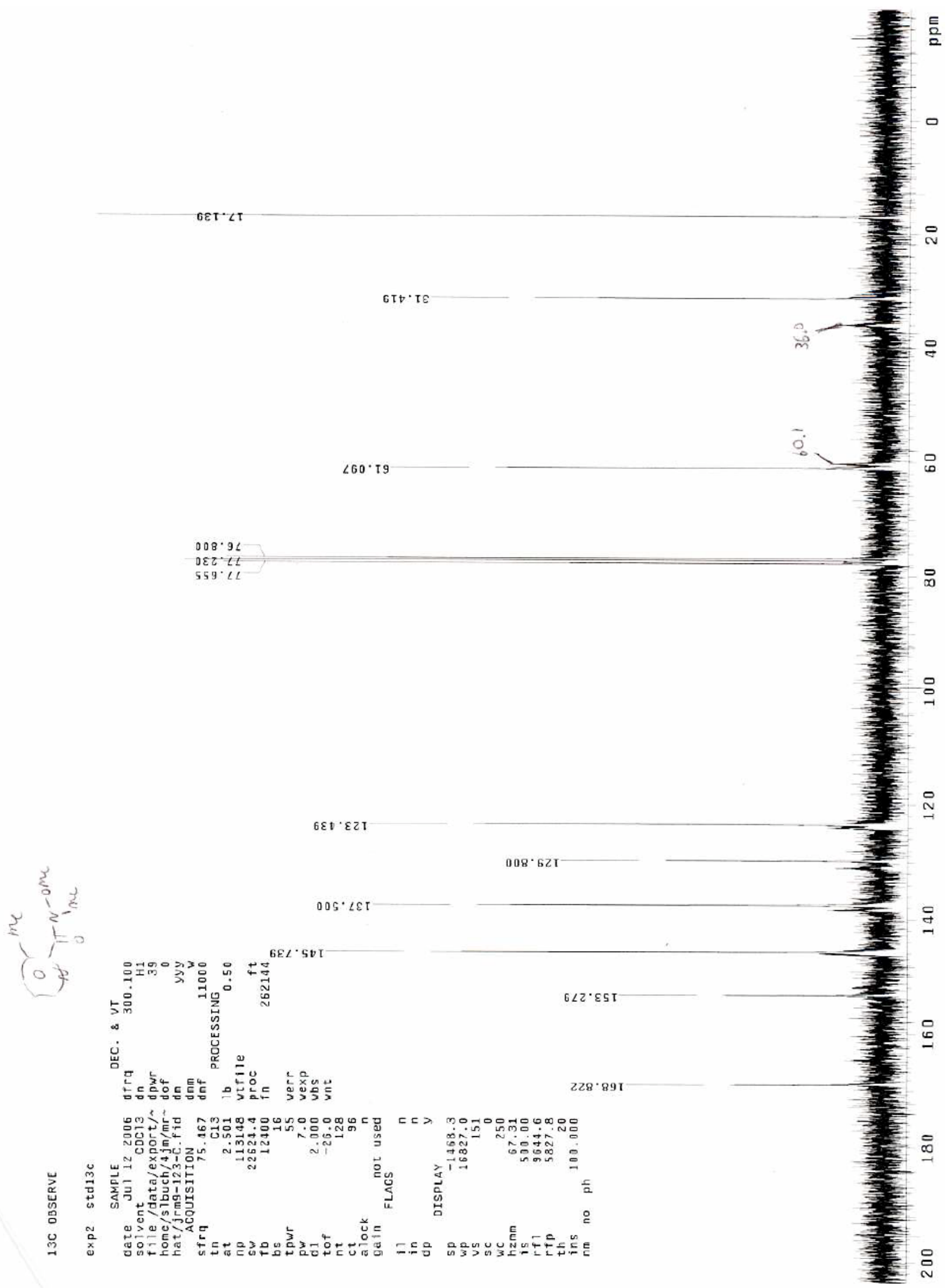




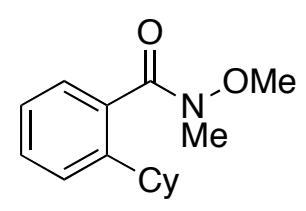

2-Cyclohexyl- $N$-methoxy- $N$-methyl-benzamide (Table 3, entry 9). Following general procedure $\mathrm{B}$, a mixture of 1-bromo-2-cyclohexylbenzene $(1 \mathrm{mmol}, 0.239 \mathrm{~g}), \mathrm{Pd}(\mathrm{OAc})_{2}(3.0 \mathrm{~mol} \%, 0.03 \mathrm{mmol}, 0.03$ equiv., $6.7 \mathrm{mg})$, Xantphos (6 mol \%, $0.06 \mathrm{mmol}, 0.06$ equiv., $34.7 \mathrm{mg}$ ), $N, O-$ dimethylhydroxylamine hydrochloride $(1.5 \mathrm{mmol}, 1.5$ equiv., $146 \mathrm{mg}), \mathrm{K}-$ ${ }_{3} \mathrm{PO}_{4}(3 \mathrm{mmol}, 3$ equiv., $637 \mathrm{mg})$, and $m$-xylene $(2 \mathrm{~mL})$ was heated at $120{ }^{\circ} \mathrm{C}$ for 20 hours. The crude product mixture was purified by flash column chromatography on silica gel (30 - 50\% ethyl acetate in hexanes) to provide a mixture of rotamers (ratio not determined) of the title

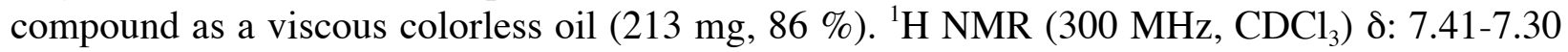
(m, 2H), 7.24-7.14 (m, 2H), 3.85 (brs, 1H), 3.38 (brs, 5H), 2.75 (brs, 1H), 1.60-1.95 (m, 5H),

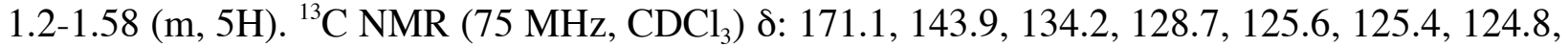
60.2, 41.0, 33.6, 31.6, 26.3, 25.5. IR (neat, $\left.\mathrm{cm}^{-1}\right): 3292,3061,3025,2926,2851,2817,2668$, $1651,1599,1575,1489,1448,1410,1378,1264,1218,1193,1168,1140,1117,1093,1060$, $1044,989,893,884,863,829,771,755,705,644,634,625,577,530$. Anal. Calcd for $\mathrm{C}_{15} \mathrm{H}_{21} \mathrm{NO}_{2}$ : C, 72.84; H, 8.56. Found: C, 72.66; H 8.54.

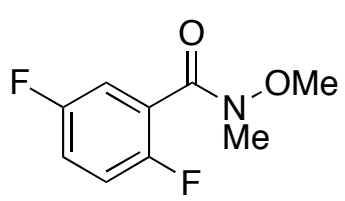

2,5-Difluoro- $N$-methoxy- $N$-methyl-benzamide (Table 3, entry 10). Following general procedure $\mathrm{B}$, a mixture of 2,5-difluorobromobenzene ( $1 \mathrm{mmol}, 0.193 \mathrm{~g}$, filtered through basic alumina prior to use), $\mathrm{Pd}(\mathrm{OAc})_{2}$ ( $2.5 \mathrm{~mol} \%, 0.025 \mathrm{mmol}, 0.025$ equiv., $5.6 \mathrm{mg}$ ), Xantphos ( $5 \mathrm{~mol} \%, 0.05$ mmol, 0.05 equiv., $28.9 \mathrm{mg}$ ), $N$, $O$-dimethylhydroxylamine hydrochloride (1.5 mmol, 1.5 equiv., $146 \mathrm{mg}), \mathrm{K}_{3} \mathrm{PO}_{4}(3 \mathrm{mmol}, 3$ equiv., $637 \mathrm{mg})$, and $m$-xylene $(2 \mathrm{~mL})$ was heated at $110{ }^{\circ} \mathrm{C}$ for 20 hours. The crude product mixture was purified by flash column chromatography on silica gel (50\% ethyl acetate in hexanes) to provide the title compound as a colorless oil (140 mg, $70 \%$ ). ${ }^{1} \mathrm{H}$ NMR $\left(300 \mathrm{MHz}, \mathrm{CDCl}_{3}\right.$ ) $\delta$ : 7.19-7.03 (m, 3H), 3.56 (brs, $\left.3 \mathrm{H}\right)$, 3.36 (brs, $3 \mathrm{H}$ ). ${ }^{13} \mathrm{C}$ NMR $\left(75 \mathrm{MHz}, \mathrm{CDCl}_{3}\right.$ ) $\delta: 164.7,159.7,156.4,156.1,152.9,124.7,124.6$, 124.4, 124.3, 117.9, 117.6, 117.1, 117.0, 116.8, 116.7, 115.4, 115.1, 61.1, 31.9 (observed complexity due to C-F splitting; definitive assignments have not yet been made). ${ }^{19} \mathrm{~F}$ NMR (282 $\mathrm{MHz}, \mathrm{CDCl}_{3}$ ) $\delta:-118.8,-120.4$. IR (neat, $\mathrm{cm}^{-1}$ ): 3074, 2977, 2940, 2823, 1659, 1599, 1495, $1437,1405,1383,1266,1251,1205,1149,1104,1059,992,939,879,851,822,786,735,706$, 690, 640, 604. Anal. Calcd for $\mathrm{C}_{9} \mathrm{H}_{9} \mathrm{~F}_{2} \mathrm{NO}_{2}: \mathrm{C}, 53.73 ; \mathrm{H}, 4.51$. Found: C, 53.50; H, 4.55.

\section{References}

${ }^{1}$ (a) Pangborn, A. B.; Giardello, M. A.; Grubbs, R. H.; Rosen, R. K.; Timmers, F. J. Organometallics 1996, 15, 1518. (b) Alaimo, P. J.; Peters, D. W.; Arnold, J.; Bergman, R. G. J. Chem. Ed. 2001, 78, 64.

2 (a)Seganish, W. M.; DeShong, P. J. Org. Chem. 2004, 64, 6790. (b) Deetz, M. J.; Forbes, C. C.; Jonas, M.; Malerich, J. P.; Smith, B. D.; Wiest, S. J. Org. Chem. 2002, 67, 3949.

${ }^{3}$ De Voss, J. J.; Sui, Z.; DeCamp, D. L.; Salto, R.; Babé, L. M.; Craik, C. S.; Ortiz de Montellano, P. R. J. Med. Chem. 1994, 37, 665.

${ }^{4}$ Einhorn, J.; Einhorn, C.; Luche, J.-L. Synth. Commun. 1990, 20, 1105. 